\title{
EXPERIMENTAL TESTING, MODELING, AND SIMULATION OF 3D PRINTED COMPOSITE MATERIAL FOR MORPHING WING APPLICATION
}

\author{
Rebecca Rajs ${ }^{1}$, Marc Palardy-Sim²*, Guillaume Renaud ${ }^{2}$, Michael Jakubinek ${ }^{2}$, Farjad Shadmehri ${ }^{1 * *}$ \\ ${ }^{1}$ Department of Mechanical, Industrial and Aerospace Engineering, Concordia University, Montreal, Quebec, Canada \\ ${ }^{2}$ National Research Council Canada, Ottawa, Ontario, Canada \\ *Marc.Palardy-Sim@cnrc-nrc.gc.ca, ${ }^{* *}$ farjad.shadmehri@concordia.ca
}

\begin{abstract}
Morphing aircraft structures offer opportunities for the development of new aerospace technologies. A benchtopscale model of a morphed leading edge composed of a carbon nanotube-polyurethane stretchable skin and 3D printed substructure was designed and developed [1]. To improve the overall accuracy of the leading edge shape, the design of the sub-structure is to be optimized. This paper describes the material characterization of the 3D printed sub-structure. The properties of the sub-structure material were determined through flexural testing of 3D printed coupons. The material properties were then calibrated through finite element modeling of the test. Finally, these properties were applied to the model of a test specimen of variable thickness in order to validate their applicability for finite element analysis of increasingly complex shapes, such as those found in the morphed leading edge benchtop model.
\end{abstract}

\section{INTRODUCTION}

Adaptive and morphing aerodynamic surfaces are an emerging design approach used to improve aircraft performance and efficiency. A number of studies have focused on the leading- and trailing-edge of aircraft wings, demonstrating the improved performance achievable [1-7]. While designs involving compliant, flexible structures are more common, stretchable designs for the leading edge of the wing would allow for significant area change leading to improved aerodynamic efficiency $[2,3]$. For example, a drooped leading edge with a net area change up to $20 \%$ has the potential to substantially increase the maximum lift coefficient compared to an unstretched leading edge [2]. However, implementation of such a morphing wing presents various technical issues. It must be possible to vary the leading edge shape while maintaining a smooth and continuous aerodynamic profile. Furthermore, the structure must enable large-scale deformation, and the corresponding area increase, while still being lightweight and maintaining enough rigidity to withstand aerodynamic loads.

The National Research Council of Canada (NRC) developed a benchtop prototype of a stretchable morphing leading edge concept [1]. The prototype consists of three components: a multifunctional nanocomposite skin, a 3D printed compliant structure to support the skin and maintain the airfoil shape, and a rigid strut structure to support and guide the leading edge structure (Fig. 1).

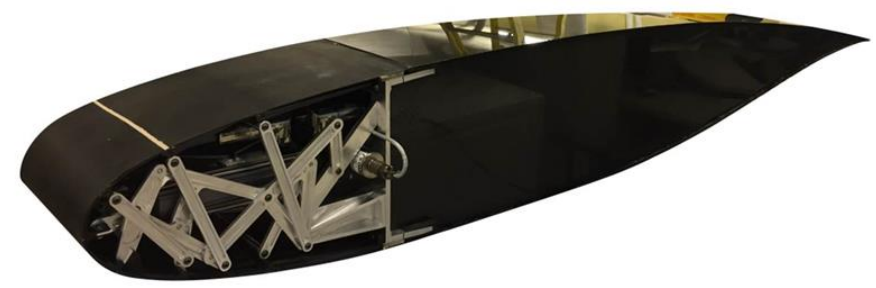

Figure 1. Bench model, including the stretchable leading edge and a fixed trailing edge. Reproduced from [1] with permission.

However, while the initial (cruise) shape closely resembles the target shape, the droop-shape as shown in Fig. 2 deviates from the target shape and the support substructure design requires further optimization to improve the accuracy and smoothness of the leading edge shape in this configuration. In order to do so, the material properties and behavior of the substructure, as well as the interaction between the substructure and the skin, must be properly characterized. Here, the experimental characterization of the material properties of the 3D printed support structure is described, as well as the calibration of a finite element (FE) model to produce matching simulation results.
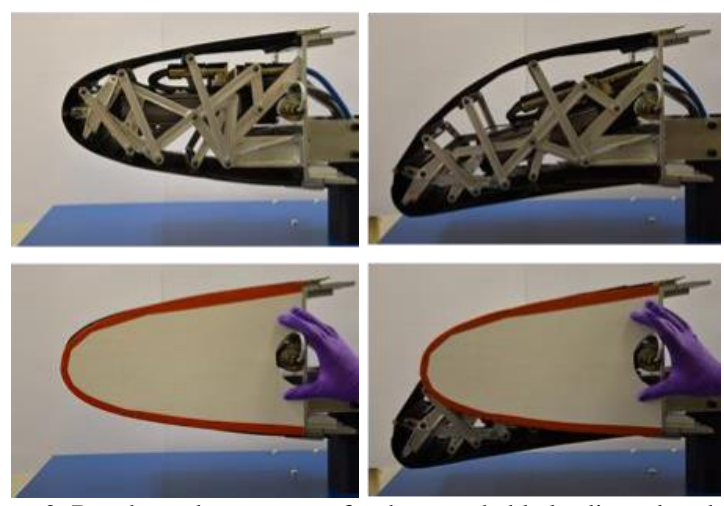

Figure 2. Benchtest demonstrator for the stretchable leading edge shown during morphing from cruise (left) to droop (right) along with a comparison to the targeted cruise shape. Reproduced from [1] with permission 


\section{MATERIAL CHARACTERIZATION AND COUPON TESTING}

\section{A. Test Samples and Experimenatal Setup}

The morphing wing substructure elements were printed using a Mark Two printer (MarkForged) and chopped-carbon fiber-reinforced Nylon filament (Onyx, MarkForged). In order to properly model the material in the finite element model, a 3point bending test was first conducted to determine the effective flexural properties of the material. Coupons measuring $127 \mathrm{~mm}$ in length, $12.7 \mathrm{~mm}$ in width, and $3.3 \mathrm{~mm}$ in depth were printed using the maximum fill factor with three different fill patterns: solid, rectangular, and triangular. Following the ASTM D790-17 standard for flexural testing of plastics, three coupons of each fill pattern were tested at deflections ranging from $2 \mathrm{~mm}$ to $10 \mathrm{~mm}$ and a deflection rate of $13.7 \mathrm{~mm} / \mathrm{min}$ [8]. Data points were collected every 100 milliseconds. A small preload was applied to the coupon on the test fixture to ensure that there was sufficient contact between the crosshead and the coupon surface prior to testing each sample. All testing was performed using an MTS Criterion 41 electro-mechanical load frame.

The obtained crosshead displacement versus load data was averaged for each fill pattern, smoothed using curve fitting to reduce any noise in the raw data, and zeroed. From the averaged and adjusted experimental data, the flexural stress and strain were calculated at each data point throughout the deflection of the coupon using equations (1) and (2),

$$
\begin{gathered}
\sigma_{f}=3 P L / 2 b d^{2} \text { and } \\
\varepsilon_{f}=6 D d / L^{2}
\end{gathered}
$$

where:

$\sigma=$ stress $(\mathrm{MPa})$ in the outer layers at midpoint,

$\varepsilon_{f}=$ strain in the outer surface,

$P=\operatorname{load}(\mathrm{N})$ at a given point on the load-deflection curve,

$L=$ support span (mm),

$b=$ width $(\mathrm{mm})$ of beam tested,

$d=$ depth $(\mathrm{mm})$ of beam tested, and

$D=$ maximum deflection $(\mathrm{mm})$ of the center of the beam.

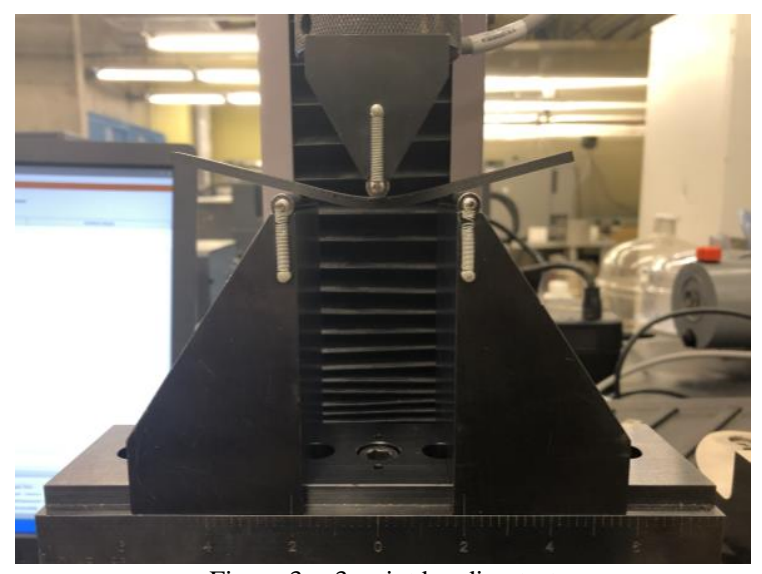

Figure 3. 3-point bending test

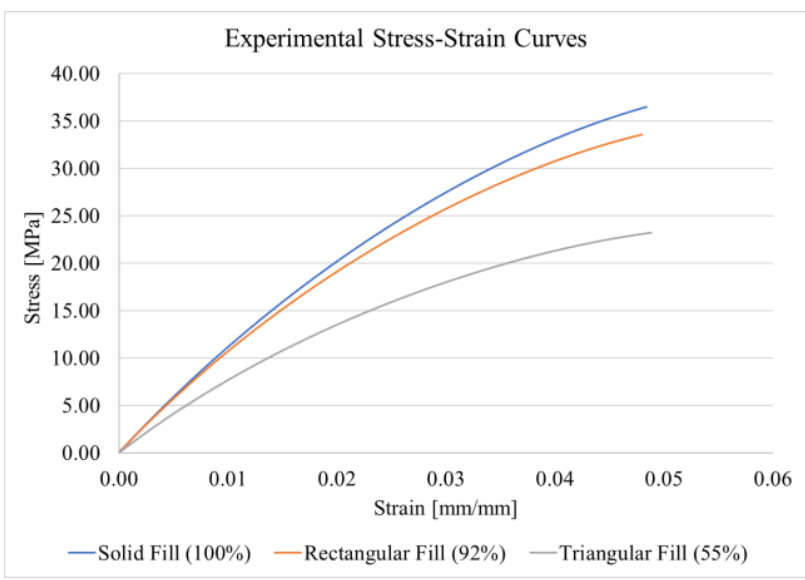

Figure 4. Experimental stress-strain curves for different fill patterns

Fig. 3 illustrates the 3-point bending test of the coupons. The experimental setup consisted of the coupon resting on support rollers, at a span of $50.4 \mathrm{~mm}$, being deflected by the crosshead roller. Fig. 4 shows the experimentally obtained stress-strain curves for each fill type. These curves represent only the loading portion of the experiment. This is because the samples exhibited some deformation once they were unloaded. This deformation was not permanent, and the samples returned to their original flat shapes after some time. The higher density fill patterns produced stiffer samples with larger values of stress for a given value of strain. The material exhibited linear behavior at the beginning followed by nonlinear behavior after about $0.8 \%$ strain regardless of fill pattern.

\section{B. Finite Element Modeling and Simulation}

The test setup shown previously in Fig. 3 was replicated using a nonlinear FE model. The model consisted of a coupon in contact with two supports. The model was developed using the Nastran/Patran commercial package. The coupon was modelled using thin 2D shell elements having constant thickness. Nonlinear properties in thin 2D shell elements are formulated following linear discrete Kirchhoff integration which neglects transverse shear deformation. The length of the model was divided into 60 elements, and the width into 10 elements. A mesh convergence study was performed to ensure these numbers of elements were sufficient for the analysis. Nonlinear elastic model was selected as the constitutive model in the FE software. A three-dimensional representation of the model is shown in Fig. 5. The experimental stress-strain curves from Fig. 4 were used as inputs for the material properties in the model.

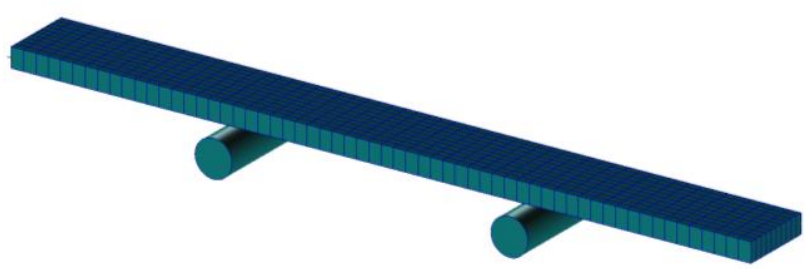

Figure 5. FE model of the coupon (3D representation) 
The FE model was run using the implicit nonlinear solution (SOL400) offered by Nastran/Patran commercial package. The material was assumed non-linear elastic and viscous effects were neglected. As such, strain-rate effects were not considered. This assumption was considered reasonable for the loading phase, which was done at a low rate. Due to the nonlinearity of the material properties, there was some error introduced in the calculation of the stress using equation (1), which assumes that the material exhibits a linear relationship between stress and strain up to the point of rupture. To account for this error, the stress-strain curve was adjusted through a linear reduction of the force based on the error at each iteration. For example, in the first iteration, at a deflection of $2 \mathrm{~mm}$, the experimentally obtained force was approximately $28.9 \mathrm{~N}$. However, the FE simulation predicted a force of $31.5 \mathrm{~N}$. Therefore, the stress at this point was reduced by $5.4 \%$. This reduction was applied at each point on the stress-strain curve until a new adjusted curve was found, which was then used as the input to the material model in the FE simulation. This process was repeated until the results from the simulation coincided with the experimental results within $2 \%$.

The results of each iteration for the solid fill pattern can be seen in Fig. 6, where after four iterations the FE analysis agreed with the experimental results. This final adjusted stressstrain curve represents the calibrated flexural properties of the 3D printed material.

\section{APPLICATION TO VARIABLE THICKNESS BEAM}

\section{A. Morphing Wing Substructure Design}

In order to maintain the curvature of the leading edge of the morphing wing in both the cruise and drooped shapes, a series of interleaved variable thickness beam structures (Fig. 7) were employed. These beams had various thicknesses and lengths, depending on where they were located along the leading edge. They were designed analytically and then verified using a nonlinear FE model. However, as described previously, there were significant deviations between the previous iteration of the FE model and the final benchtest deformed shape as shown in Fig. 3.

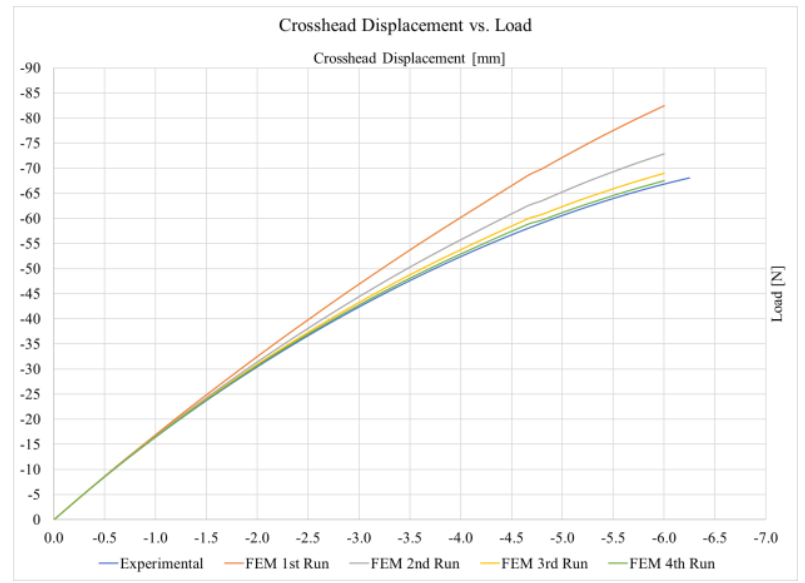

Figure 6. FE analysis results for solid fill coupons

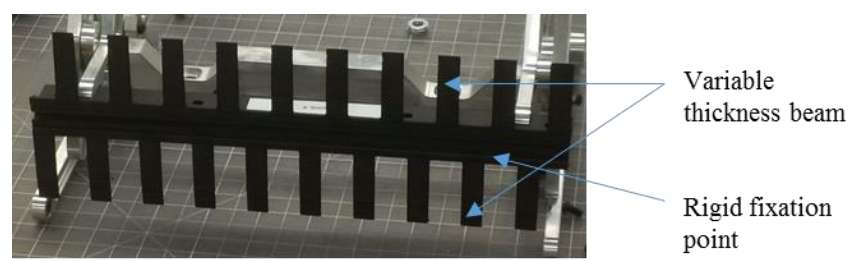

Figure 7. A single section of the substructure consisting of interleaving variable thickness beams protruding from a rigid fixation point.

\section{B. Test Samples and Experimental Setup}

In order to validate the applicability of the adjusted material model for the morphing wing sub-structure application, a second set of tests was conducted on a simplified variable thickness beam sample. The geometry of the variable thickness sample can be seen in Fig. 8 and the sample was printed using the solid fill pattern (100\% fill). A 3-point bending test was performed using variable support spans of $101.6 \mathrm{~mm}, 127 \mathrm{~mm}$, and $152.4 \mathrm{~mm}$, and crosshead deflections ranging from $2 \mathrm{~mm}$ to $10 \mathrm{~mm}$ and a deflection rate of $13.7 \mathrm{~mm} / \mathrm{min}$. Data points were collected every 100 milliseconds. An example of the test setup can be seen in Fig. 9 for a $6 \mathrm{~mm}$ deflection at a span of $101.6 \mathrm{~mm}$. The resulting crosshead displacement versus load curves for each support span and deflection were then found. As with the coupon tests, a small preload was applied to the sample to ensure proper contact with the crosshead. Therefore, the crosshead displacement versus load curves were zeroed prior to comparing them with the results from the FE model.

\section{Finite Element Modeling and Simulation}

Nonlinear FE models of the test setup in Fig. 9 were created for each support span. The comb was modelled using thin 2D shell elements having variable thickness. Shell elements were used in order to maintain consistency with the FE model of the coupon tests. Also, using shell elements for the modeling of the entire leading edge of the morphing wing will reduce solving time and overall complexity. Based on a mesh convergence study, the model had 80 elements in length, and 20 elements in width. The final adjusted stress-strain curve found from the coupon tests in Section II was used as the input for the model material properties.

The FE model was run using the implicit nonlinear solution (SOL400) offered by Nastran/Patran commercial package. Initial analyses with the model exhibited behavior stiffer than experimentally observed. Adjustments to the model, as described below, were made until the results agreed with those obtained experimentally. The final model for the $101.6 \mathrm{~mm}$ span tests can be seen in Fig. 10. As can be seen, the thickness of the center portion of the comb was reduced in the model.

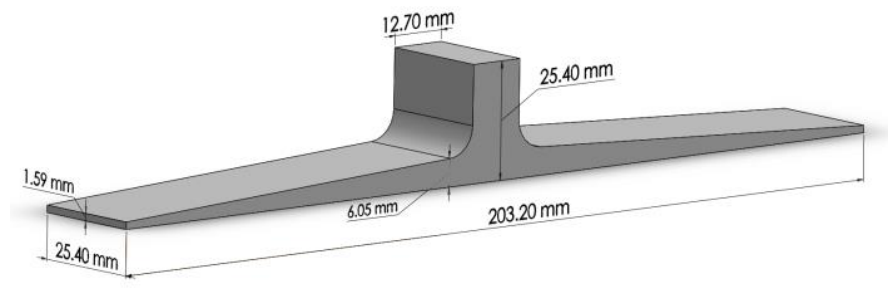

Figure 8. Simplified variable-thickness beam design for model validation. 


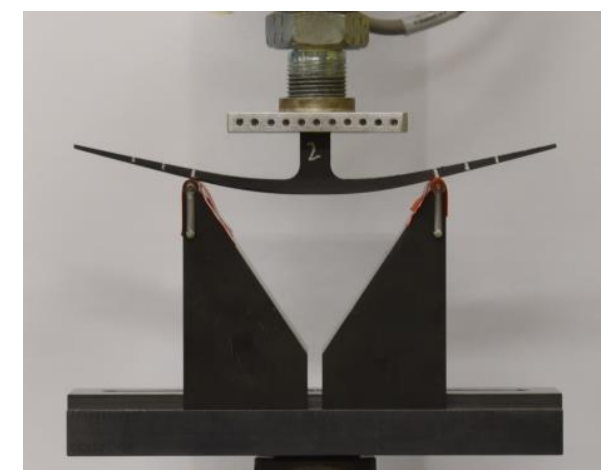

Figure 9. 3-Point bending test of a variable-thickness comb

This was done to reduce the stiffening effect it had on the resulting forces from the FE model simulation results. The thickness was gradually reduced until the outputs of the FE models at all 3 support spans were within $15 \%$ of the experimental results at the maximum deflection. This was achieved by reducing the thickness from $25.4 \mathrm{~mm}$ to $6.25 \mathrm{~mm}$.

Results of the simulation for each support span at a deflection of $6 \mathrm{~mm}$ can be seen in Fig. 11. The force required to deflect the comb increased with decreasing span length. Excellent agreement between the experimental results and those of the FE simulation was achieved as can been in Fig. 11. At deflections below $1 \mathrm{~mm}$, there is a $3 \%$ average difference in the force results. There is, on average, a $7.7 \%$ difference in force between the experimental results and those of the FE simulation at the largest displacement.

Some degree of error is present in the results for the variable thickness beam due to the fact that only one sample was used. This sample was tested multiple times, and therefore it exhibited some slight variation in shape after each test run due to viscoelastic behavior of the material and the large amount of relaxation time required for the beam to fully return to its original shape. The beam was also not perfectly flat due to $3 \mathrm{D}$ printing process and had a slight natural upwards curvature before the testing began, which was not captured in the FE model.

An example of the deformed shape comparison is shown in Fig. 13. Overall, the FE deflection curve agrees well with the experimental result. It can be seen that the FE model exhibits approximately $25 \%$ less deflection at the extremities of the comb compared to the actual sample. However, the shape between the two supports is reflected accurately in the FE model. These differences can be attributed to the causes mentioned above. Overall, the results show that it is possible to generate an FE model using the material properties derived from the coupon tests which matches the behavior of a variable thickness comb.

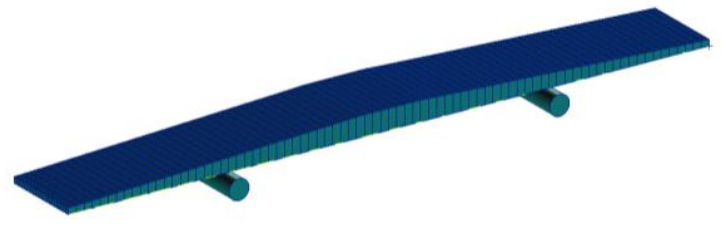

Figure 10. FE Model of the Variable Thickness Comb at 101.6mm Span (3D representation)

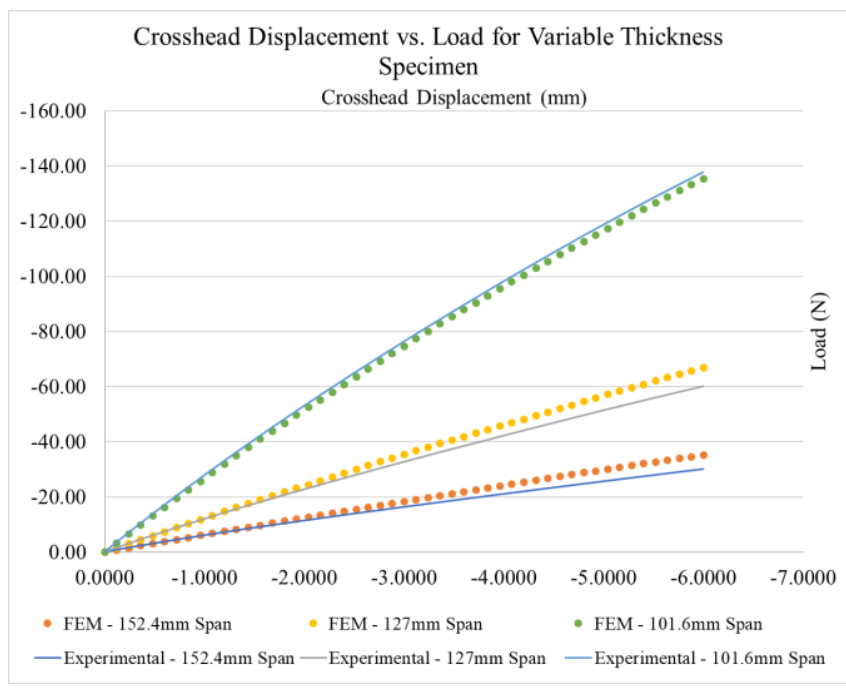

Figure 11. FE results for variable-thickness comb at various support spans

\section{CONLUSION}

The ongoing activities towards the optimization of the substructure design of a stretchable morphed leading edge were described. Flexural testing of 3D printed coupons allowed for the determination of the sub-structure material properties. Using a nonlinear FE model of the flexural test setup, the material properties were adjusted and calibrated to produce matching results. A variable thickness beam specimen was then tested and modelled using the adjusted material properties. The results show that, the adjusted material properties can be applied to specimens of varying thickness and size, allowing for the modeling of more complex comb designs using 2D shell elements, which is required for the optimization of the morphed leading edge sub-structure design. Further testing includes the addition of the nanocomposite skin coupled with 3D printed combs to properly model the interaction between the skin and substructure, and their combined behavior. Once this behavior is properly captured, optimization can be done on the comb designs found in the morphing wing to improve the accuracy and smoothness of the leading edge shape in the droop configuration.

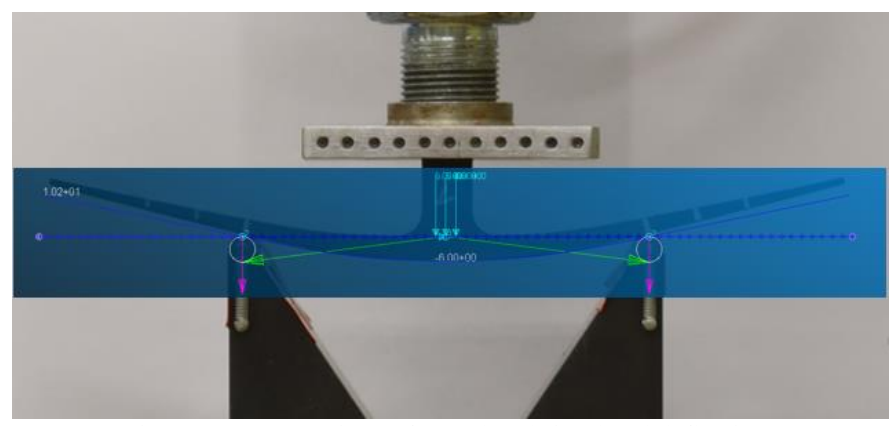

Figure 13. Comparison of actual and simulated deflections 


\section{ACKNOWLEDGMENT}

Support from Concordia Undergraduate Student Research Award (CUSRA) and research grant number V01292 is appreciated. The authors also acknowledge funding from the National Research Council Canada for development of the morphing wing demonstration and thank the Mission Optimized Smart Structures Project team, led by Dr. Wajid Chishty at NRC.

\section{REFERENCES}

[1] M. Jakubinek, S. Roy, M. Palardy-Sim, B. Ashrafi, F. Shadmehri, G. Renaud, M. Barnes, Y. Martinez-Rubi, M. Rahmat, B. Simard, A. Yousefpour, F. Fortin. Stretchable structure for a benchtop-scale morphed leading edge demonstration. AIAA SciTech 2019-1857, doi: 10.2514/6.2019-1857 (2019).

[2] F. Fortin, G. Renaud, S. Roy. Shape optimization of a stretchable drooping leading edge. AIAA SciTech 2019-2352, doi: 10.2514/6.20192352 (2019).

[3] P. Gamboa, J. Vale, F.J.P. Lau, A. Suleman. Optimization of a morphing wing based on coupled aerodynamic and structural constraints. AIAA Journal 47, September 2009.

[4] Sinapius, M., et al. "DLR's morphing wing activities within the European network." Mechanics 2210.9838 (2013).

[5] Kota, Sridhar, et al. "Design and application of compliant mechanisms for morphing aircraft structures." Smart structures and materials 2003: industrial and commercial applications of smart structures technologies. Vol. 5054. International Society for Optics and Photonics, 2003.

[6] S. Vasista, J. Riemenschneider, R. Keimer, H.P. Monner, F. Nolte, P. Horst. Morping wing droop nose with large deformation: ground tests and lessons learned. Aerospace 6, 111 (2019).

[7] S. Barbarino, O. Bilgen, R.M. Ajaj, M.I. Friswell, D.J. Inman. A review of morphing aircraft. Journal of Intelligent Material Systems and Structures 22, 823 (2011)

[8] Standard Test Methods for Flexural Properties of Unreinforced and Reinforced Plastics and Electrical Insulating Materials, ASTM Standard D790, 2017 